\title{
Indolent lymphomas: introduction to a series highlighting progress and ongoing challenges
}

\author{
Sonali M. Smith ${ }^{1}$ and Gilles Salles ${ }^{2}$
}

${ }^{1}$ Section of Hematology/Oncology, The University of Chicago, Chicago, IL and ${ }^{2}$ Memorial Sloan Kettering Cancer Center, New York, NY, USA

Haematologica 2022

Volume 107(1):4-6

\section{Correspondence:}

SONALI M. SMITH

smsmith@medicine.bsd.uchicago.edu

Received: October 19, 2021.

Accepted: October 19, 2021.

https://doi.org/10.3324/haematol.2021.280218

(C)2022 Ferrata Storti Foundation

Material published in Haematologica is covered by copyright. All rights are reserved to the Ferrata Storti Foundation. Use of published material is allowed under the following terms and conditions:

https://creativecommons.org/licenses/by-nc/4.0/legalcode. Copies of published material are allowed for personal or internal use. Sharing published material for non-commercial purposes is subject to the following conditions:

https://creativecommons.org/licenses/by-nc/4.0/legalcode, sect. 3. Reproducing and sharing published material for commercial purposes is not allowed without permission in writing from the publisher.

\section{Introduction}

Indolent lymphomas collectively comprise up to one-third of all non-Hodgkin lymphomas, with an ever-increasing number of survivors. Despite the indolent course, patients are subject to repeated relapses and remissions, and exposed to a recurrent need for treatment and intervention. The ultimate challenge with these lymphomas is that cure remains elusive, and the impact of the disease on quality and quantity of life is significant; a portion of patients will die from either disease or treatment-associated toxicity, and lymphoma-related mortality remains the most common cause of death. ${ }^{1}$ Furthermore, transformation to a more aggressive histology can occur, and drastically shifts the management approach and prognosis. Historically, the treatment approach was limited to chemotherapy or radiotherapy, and median survival times were less than a decade, likely reflecting intrinsic resistance of indolent biology to agents requiring high cell turnover for optimal effect. The past few years have witnessed significant advances in biological insights, targeted therapies, and prolonged survival for most patients. This issue of Haematologica contains a timely series on indolent lymphomas, with three comprehensive papers ${ }^{2-4}$ presented by experts in the field; the series is intended to provide an update on where the field is going in terms of new data and new perspectives, while also outlining challenges that remain.

\section{Follicular lymphoma}

As the first two papers highlight, sequential advances have dramatically improved survival for patients with follicular lymphoma (FL) which now borders on several decades for most individuals. We have finally come to a point at which most FL patients will die with lymphoma rather than from lymphoma. Unfortunately, determining individual prognosis at initial diagnosis is not yet feasible; as Cartron and Trotman ${ }^{2}$ discuss, prognostic indices at baseline (FLIPI, FLIPI2, M7-FLIPI, PRIMA-PI) are better suited to groups of patients rather than to individual patients. Furthermore, the type of treatment influences the robustness of these indices and further limits their applications. The authors also raise the limitations of the Groupe d'Etude des Lymphomes Folliculaires (GELF) criteria, and suggest opportunities for functional imaging with positron emission tomography and computed tomography for decision-making. Overall, we are unable to advise patients at the time of diagnosis on predicted outcome. Correcting this deficit is important for two reasons: we must identify those destined to do well, for whom minimal exposure to therapy is sufficient, and also identify patients with a shortened median survival for whom new or aggressive treatments are not only justified, but badly needed. For the former group, primum non nocere comes to mind, and watch and wait (or, as the authors elegantly state: "therapeutic abstention with dynamic observation") remains the standard of care.

At the other extreme are patients with rapid kinetic failure who are only identified after relapse, with two recently defined high-risk subgroups: early progression (defined as relapse within either 12 months or 24 months of initial chemoimmunotherapy) and double-refractory disease (typically defined as relapse within 6 months despite treatment with both alkylating agents and anti-CD20 monoclonal antibodies). These groups are appropriately the focus for several clinical trials, with the US Intergroup S1608 being one example of a trial specifically comparing chemo-immunotherapy against two biological doublets in patients with progression of disease within 24 months of initial chemo-immunotherapy (NCT03269669). Adding to the complexity, there is a middle group of patients, 
described by Qualls and Salles, ${ }^{3}$ in whom relapse is neither early nor yet refractory. The challenge here is that we have a myriad of options ranging from anti-CD20 antibodies alone, chemo-immunotherapy, immunomodulatory agents, phosphoinositide- 3 kinase inhibitors, an EZH2 inhibitor, transplantation and even cellular therapy. We have no data on sequencing, minimal comparative data, and no inclusion of precision approaches with the exception of better outcomes for patients with EZH2mutated FL treated with tazemetostat (as compared to patients without the EZH2 mutation). As the authors saliently note, this plethora of agents in the second line and beyond is largely marked by phase II data, gained from studies with heterogeneous inclusion criteria, and no indication on whether one treatment might preclude the use of another later on. Furthermore, although Haematologica has an international readership, it is important to acknowledge that many of the options presented will not be available to patients in the majority of the world and, even within wealthy nations, not all patients will have equal access. Qualls and Salles also highlight that newer options should not mean that the old ones have no place; as discussed in their review, radiotherapy and/or chemotherapy in the relapsed/refractory setting, while not typically preferred when alternatives are available, is valuable. An additional challenge is that many of these treatment options were designed for indefinite treatment and there is no guidance on when it is appropriate to stop treatment or consider a treatment holiday.

Extending this dilemma of an expanding toolbox without data on how to best sequence or select a specific treatment is the challenge presented by cellular therapy, or chimeric antigen receptor $\mathrm{T}$-cell therapy (CAR-T). This exciting modality engineers autologous $T$ cells to recognize CD19 on tumor cells with subsequent T-cell activation, expansion, and tumor eradication. The attendant toxicities include cytokine release syndrome and neurotoxicity, and the "financial toxicity" is considerable. Nevertheless, CAR-T has dramatically changed the algorithm for aggressive lymphomas and offers the potential for durable remission and possibly cure; its precise role in indolent lymphomas is unknown, but CAR-T with axicabtagene ciloleucel is now approved for FL. As discussed by Qualls and Salles, in the ZUMA-5 trial the response rate to CAR-T was $94 \%$ (with a complete response rate of $80 \%$ ) in heavily pretreated FL patients although the follow up is still less than 2 years. A careful review of the inclusion criteria in both the ZUMA-5 and ELARA trials shows that many patients had early progression, double refractory disease, refractory disease to the most recent treatment, and a high baseline FLIPI score., ${ }^{5,6}$ Clinicians will be asking, when should a patient with indolent lymphoma be referred for this therapy? To date, there is no consensus on this topic, but following the inclusion criteria of the published studies is reasonable.

An important aspect to furthering precision medicine in indolent lymphomas is understanding the biology and pathogenesis of the diseases, with an aim of identifying therapeutic vulnerabilities. FL is a complex and biologically heterogeneous disease, but recent insights provide valuable opportunities with several emerging themes..$^{7-9}$ First, the $t(14 ; 18)$ rearrangement, long known as a hallmark lesion, is insufficient for FL and is present in a significant portion of the normal population. Second, FL progression occurs via divergent evolution from a common progenitor cell. This critical observation may underlie the inability of our current tools to eradicate (or cure!) the disease. A reservoir population of common progenitor cells may account for the eventual resurgence of disease, even when there is clinical evidence of a complete remission. Third, sequencing of large databases of FL patients has identified epigenetic deregulation as a consistent and early event, with mutations involving KMT2D, CREBBP, $\mathrm{H} 1$ histones, EZH2, and EP300 collectively occurring in the majority of patients. Finally, FL biology and clinical behavior appear heavily reliant on the tumor microenvironment and immune composition. While these areas of FL biology under scrutiny are listed here, there are clearly other areas in active development, and the field is in an exciting phase as we contemplate the next generation of therapies.

\section{Marginal zone lymphoma}

The third paper in this series focuses on marginal zone lymphomas (MZL), a complex and heterogeneous group of diseases. MZL is a paradigmatic disease to study the role of antigenic stimuli, inflammation, and immune "irritation" leading to the development of cancer; the prototypic antigen-driven lymphoma is Helicobacter pylori-associated extranodal MZL of gastric lymphoid tissue. As described in the article by Cheah and colleagues, ${ }^{4}$ the three main clinicopathological entities have both common and distinct features. For example, deregulation of epigenetic pathways and NOTCH2 signaling are seen across multiple MZL subsets, while mutations such as PTPRD are restricted to nodal MZL. ${ }^{10}$ The treatment for MZL remains largely based on the site of clinical involvement, and is often influenced by issues of local control. A major gap in MZL is the relative paucity of disease-specific therapeutic trials; most treatment data for disseminated disease are derived from trials of FL patients. However, as many trials suggest, MZL is biologically distinct and often has disparate outcomes compared to FL; this argues for disease-specific trials and not simply lumping MZL into other trials of indolent lymphomas. An example of this observation is quite clear in cellular therapy trials in which it appears that the CAR-T approach leading to regulatory approval for FL had minimal activity in MZL. ${ }^{6}$ Despite these caveats, outcomes are generally favorable with current guidelines and a focus on short- and long-term toxicity is an essential element of the therapeutic approach. Ideally, the emerging mutational landscape and insights into the often inflammatory microenvironment will lead to more targeted therapies in the future.

\section{Concluding remarks}

When reading these reviews side-by-side, a number of common themes and struggles emerge. On a positive note, there has been dramatic progress in terms of survival and a general shift away from cytotoxic agents. A relatively new concept is that not all relapses are the same. However, several common challenges persist: inability to determine individual prognosis at the time of diagnosis, lack of data on optimal sequencing, no clear biologically driven indication for treatment selection, lim- 
ited guidance on surveillance strategies that balance risks and benefits of repeated radiological imaging, and, of course, cure remaining elusive. A clear modern-day obstacle is the ongoing COVID-19 pandemic which must be considered within the decision-making process. The negative impact of anti-lymphoma therapy on immunity puts our patients at higher risk of morbidity and mortality. There are now emerging data that rituximab or antiCD20 antibody therapy interferes with a robust antibody response to the vaccine for approximately 12 months, ${ }^{8}$ and the use of agents such as bendamustine suppresses both B- and T-cell function. The discussion on risks versus benefits of treatment and treatment selection has never been so crucial. This first series on indolent lymphomas artfully presents the progress to date in detail, with discussion of the many challenges that remain.

\section{Disclosures}

No conflicts of interest to disclose.

\section{Contributions}

The authors contributed equally.

\section{References}

1. Sarkozy C, Maurer MJ, Link BK, et al. Cause of death in follicular lymphoma in the first decade of the rituximab era: a pooled analysis of French and US cohorts. J Clin Oncol. 2019;37(2):144-152.

2. Cartron G, Trotman J. Time for an individualized approach to first-line management of follicular lymphoma. Haematologica. 2022;107(1):7-18.

3. Qualls D, Salles G. Prospects in the management of patients with follicular lymphoma beyond first-line therapy. Haematologica. 2022;107(1):19-34.

4. Cheah CY, Zucca E, Rossi D, Habermann TM. Marginal zone lymphoma: present status and future perspectives. Haematologica. 2022;107(1):35-43
5. Schuster SJ, Dickinson MJ, Dreyling M, et al. Efficacy and safety of tisagenlecleucel (Tisa-Cel) in adult patients (Pts) with relapsed/refractory follicular lymphoma $(\mathrm{r} / \mathrm{r} \mathrm{FL})$ : primary analysis of the phase 2 Elara trial. J Clin Oncol. 2021;39(15_ Suppl):7508.

6. Jacobson CA, Chavez JC, Sehgal AR, et al. Primary analysis of Zuma-5: a phase 2 study of axicabtagene ciloleucel (Axi-Cel) in patients with relapsed/refractory (R/R) indolent non-Hodgkin lymphoma (iNHL). Blood. 2020;136(Suppl_1):40-41.

7. Roulland S, Faroudi M, Mamessier E, Sungalee S, Salles G, Nadel B. Early steps of follicular lymphoma pathogenesis. Adv Immunol. 2011;111:1-46.

8. Pasqualucci L. Molecular pathogenesis of germinal center-derived B cell lymphomas.
Immunol Rev. 2019;288(1):240-261.

9. Kumar E, Pickard L, Okosun J. Pathogenesis of follicular lymphoma: genetics to the microenvironment to clinical translation. $\mathrm{Br}$ J Haematol. 2021;194(5):810-821.

10. Vela V, Juskevicius D, Dirnhofer S, Menter T, Tzankov A. Mutational landscape of marginal zone B-cell lymphomas of various origin: organotypic alterations and diagnostic potential for assignment of organ origin. Virchows Arch. 2021;Sep 8. [Epub ahead of print].

11. Gurion R, Rozovski U, Itchaki G, et al. Humoral serologic response to the BNT162b2 vaccine is abrogated in lymphoma patients within the first 12 months following treatment with anti-CD2O antibodies. Haematologica. 2021;Jul 29. [Epub ahead of print]. 